\title{
Interaction of magnetic vortices with defects
}

\author{
A. S. Kovalev, and J. E. Prilepskii
}

Citation: Low Temperature Physics 44, 663 (2018); doi: 10.1063/1.5041432

View online: https://doi.org/10.1063/1.5041432

View Table of Contents: http://aip.scitation.org/toc/ttp/44/7

Published by the American Institute of Physics

\section{Articles you may be interested in}

Localization of magnon modes in a curved magnetic nanowire

Low Temperature Physics 44, 634 (2018); 10.1063/1.5041428

Bullets and droplets: Two-dimensional spin-wave solitons in modern magnonics (Review Article)

Low Temperature Physics 44, 602 (2018); 10.1063/1.5041426

Electric control of cooperative polariton dynamics in a cavity-magnon system

Applied Physics Letters 112, 262401 (2018); 10.1063/1.5024336

Thermal tuning of silicon terahertz whispering-gallery mode resonators

Applied Physics Letters 113, 011101 (2018); 10.1063/1.5036539

Arnold Markovich Kosevich (1928-2006): On the occasion of his 90th birthday

Low Temperature Physics 44, 599 (2018); 10.1063/1.5041425

Dynamic solitons in antiferromagnets (Review Article)

Low Temperature Physics 44, 618 (2018); 10.1063/1.5041427

LOW TEMPERATURE TECHNIQUES

OP『|CAL CAVI『Y PHYSICS

MITIGATING THERMAL

\& VIBRATIONAL NOISE

DOWNLOAD THE WHITE PAPER 


\title{
Interaction of magnetic vortices with defects
}

\author{
A. S. Kovalev ${ }^{\text {a) }}$
}

B.I. Verkin Institute for Low Temperature Physics and Engineering, National Academy of Sciences of Ukraine, 47 Nauki Prospect, Kharkiv 61103, Ukraine and V.I. Karazin Kharkiv National University, 4 Svobody Square, Kharkiv 610022, Ukraine

\section{J. E. Prilepskii}

Aston University, Birmingham, UK

(Submitted March 2, 2018)

Fiz. Nizk. Temp. 44, 847-856 (July 2018)

\begin{abstract}
Scattering of magnetic vortices and vortex pairs by a magnetic defect in two-dimensional easy-plane ferromagnets was theoretically studied using classical equations of magnetization dynamics and the approximation of collective variables for the coordinates of vortices. A defect model was proposed as a local region of a magnet whose exchange interaction differs from that of the remaining part. The rotation of the magnetic vortex around the defect and the associated scattering of vortex pairs (vortex-antivortex bound states) were considered. In the limit of small-angle scattering, analytical expressions were obtained for the differential scattering cross section and the dependence of the scattering angle on the parameters of the vortex pair, defect and impact distance. The scattering of vortex pairs by a defect was studied, numerically and qualitatively, in the entire range of scattering parameters. Our study indicated that the impact parameter has critical values at which the scattering pattern changes in a qualitative manner and defect-localized rotational states of vortex pairs possibly exist. Published by AIP Publishing. https://doi.org/10.1063/1.5041432
\end{abstract}

\section{Introduction}

Important objects in the study of nonlinear magnet dynamics include solitons of various types, and in particular topological solitons ${ }^{1,2}$ which may be represented, for example, by vortices in easy-plane ferromagnets. ${ }^{3,4}$ The study of vortex excitations in condensed systems is representative of the traditional field of hydrodynamics, dynamics of superflu$\operatorname{ids}^{5}$ and, recently, studies of Bose-Einstein condensation (BEC). ${ }^{6}$ Interest in magnetic vortices has recently arisen due to their possible use in spintronics. ${ }^{7}$ The unfavorable aspects, however, include a low displacement rate of vortices and their strong interaction with impurities. In this regard, vortex pairs capable of displacement at velocities on the order of the magnon velocity and excitation by various methods are promising. ${ }^{8}$ This, however, gives rise to an important problem of the interaction of vortex pairs with defects in the magnetic structure. The dynamics and interaction of magnetic vortex pairs has been widely discussed in theoretical terms. $^{9,10}$ Less is known about the interaction of isolated vortices with defects, for which contradictory results have been obtained. ${ }^{11,12}$ The dynamics of vortex pairs in the area of a defect is more complex, as can be judged from the numerical analysis of this problem in BEC. ${ }^{13}$ This paper addresses the interaction of magnetic vortices and vortex pairs with a defect within the defect model described herein.

\section{Model formulation}

A ferromagnet with an isotropic easy-plane magnetic anisotropy in the classical approach is characterized by energy $^{1,2}$

$$
E=\int \varepsilon d x d y=\int\left(J a_{0}^{2}(\nabla \mathbf{M})^{2} / 2+\beta M_{z}^{2} / 2\right) d x d y,
$$

where $\mathbf{M}$ is the magnetic moment; $J, \beta$ are the constants of exchange interaction and single-ion anisotropy $(\beta>0)$ associated with the $Z$ axis perpendicular to the easy plane $X Y$. The dynamics of the vector $\mathbf{M}$ may be described by the Landau-Lifshitz equation (LLE) ${ }^{14}$

$$
\partial \mathbf{M} / \partial t=\left(2 \mu_{0} / \hbar\right)[\mathbf{M}, \delta \varepsilon / \delta \mathbf{M}]
$$

where $\mu_{0}$ is the Bohr magneton. It is convenient to set the $Z$-component of the moment $m=M_{z} / M_{0}$ and the azimuthal angle of its rotation in the $X Y$ plane $\varphi=\operatorname{arctg}\left(M_{y} / M_{x}\right.$ as variables, which play the role of canonically conjugated quantities, in terms of which the Hamiltonian equations and energy (1) are given by

$$
\begin{aligned}
\dot{\varphi} & =-\left(2 \mu_{0} / \hbar M_{0}\right) \partial \varepsilon / \partial m, \quad \dot{m}=\left(2 \mu_{0} / \hbar M_{0}\right) \partial \varepsilon / \partial \varphi \\
E= & \left(M_{0}^{2} / 2\right) \int\left(J a_{0}^{2}\left((\nabla \mathrm{m})^{2} /\left(1-m^{2}\right)+\left(1-m^{2}\right)(\nabla \varphi)^{2}\right)\right. \\
& \left.+\beta m^{2} d x d y\right) .
\end{aligned}
$$

As defects with axial symmetry are considered below, it follows that the $Z$-projection of the angular momentum $\mathbf{K}^{1,2}$ is conserved in addition to the energy

$$
\mathbf{K}=\mathbf{n}_{z}\left(\hbar M_{0} / 2 \mu_{0}\right) \int m(x(\partial \varphi / \partial y)-y(\partial \varphi / \partial x)) d x d y .
$$

The static solution of the LLE (3) for the magnetic vortex at the point $(x=X, y=Y)$ has the following form

$$
\begin{aligned}
& \varphi=q \operatorname{arctg} \frac{y-Y}{x-X}= \pm \operatorname{arctg} \frac{y-Y}{x-X} \\
& m=m(r)=p f\left(\sqrt{(x-X)^{2}+(y-Y)^{2}}\right)= \pm f,
\end{aligned}
$$


where the parameter $q= \pm 1$ determines the topological charge of the "vortex" with $q=1$ and the "antivortex" with $q=-1$, and parameter $p= \pm 1$ determines its polarization, whereas vortex magnetization $m(r)$ is localized in its core with the size $d_{0}$ of the order of "magnetic length" $l_{0}$ $=a_{0} \sqrt{J / \beta}{ }^{3,4}$ The topological properties of the vortex determine its dynamics and are characterized by the value of the gyro-vector $\mathbf{G}=-2 \pi p q\left(\hbar M_{0} / 2 \mu_{0}\right) \mathbf{n}_{z}$.

If the distances between vortices and between these and the boundaries of the magnet are much greater than the magnetic length, and the velocities of the vortices are much lower than those of magnons $c=\left(2 \mu_{0} M_{0} / \hbar\right) \sqrt{J \beta}$, the vortex dynamics can be approximately described in terms of the coordinates of vortex centers $\mathbf{R}_{i}(t)$ and the Thiele equations; ${ }^{15}$ therefore

$$
\left[\dot{\mathbf{R}}_{i}, \mathbf{G}_{i}\right]+\partial E / \partial \mathbf{R}_{i}=0
$$

and the energy of the system is reduced ${ }^{15}$ to the sum

$$
E=-2 \pi J M_{0}^{2} \sum_{i<j} p_{i} p_{j} \ln \left|\mathbf{R}_{i}-\mathbf{R}_{j}\right|
$$

In the same approximation, the angular momentum of the vortex system equals

$$
K=\left(\pi \hbar M_{0} / 2 \mu_{0}\right) \sum_{i} p_{i} q_{i} \mathbf{R}_{i}^{2}
$$

Equation (7) can be given a somewhat different and sometimes more convenient form ${ }^{4}$

$$
\dot{\mathbf{R}}_{i}=-p_{i} \nabla \varphi_{\mathrm{ex}}\left(\mathbf{r}=\mathbf{R}_{i}\right)\left(2 \mu_{0} M_{0} / \hbar\right),
$$

where $\varphi_{\mathrm{ex}}$ is the distribution of the magnetization field in the easy plane at the location of the $i$-th vortex, caused by all the remaining vortices. The vortex has the energy $E_{0}=\pi J M_{0}^{2}$ $\ln \left(l / d_{0}\right)$, where $l$ is the distance to the nearest boundary of the magnet, as well as the intrinsic angular moment $K_{0}$ $=\hbar M p q / 2 \mu_{0}$, where $M \sim M_{0} d_{0}^{2}$ is its magnetization. The dynamics of vortex pairs and their interaction are presented a detailed analysis in Refs. 9 and 10. A vortex pair can move with velocities $V<c$, and its energy and momentum depend on the distance $L$ between the vortices and are determined by the following formulas for the case when $V \ll c$

$$
\begin{aligned}
& E=2 \pi J M_{0}^{2} \ln \left(L / d_{0}\right), \quad P=\left(\pi \hbar M_{0} / \mu_{0}\right) L, \\
& V=d E / d P=\left(2 J \mu_{0} M_{0} / \hbar\right) / L .
\end{aligned}
$$

Let us now consider the dynamics of a vortex pair in an inhomogeneous infinite two-dimensional ferromagnet with a magnetic defect representing a radially symmetric region of radius $a$ with exchange interaction $J_{2}$ which differs in volume from exchange $J_{1}$. The values of the magnetic moment and the magnetic anisotropy in the defect do not change. It is assumed that the defect and the surrounding matrix are connected by a "hard" magnetic interaction with an infinite exchange through the boundary $J_{s}=\infty$. Therefore, the conditions on the boundary (of circle $S$ of radius $a$ ) are represented in the form of $\left.\mathbf{M}_{1}\right|_{S}=\left.\mathbf{M}_{2}\right|_{S}$ and $J_{1} d \mathbf{M}_{1} /\left.d \mathbf{n}\right|_{S}$ $=J_{2} d \mathbf{M}_{2} /\left.d \mathbf{n}\right|_{S}$, where all the below values with index 1 refer to the matrix, and with index 2 to the defect area. From the condition for the applicability of Eqs. (7) and (10), it follows that the vortices are located at a sufficiently large distance from the boundary, where $m \ll 1$. In this case, the boundary conditions reduce to the conditions for angular variable $\varphi$

$$
\left.\varphi_{1}\right|_{S}=\left.\varphi_{2}\right|_{S}, \quad J_{1} d \varphi_{1} /\left.d n\right|_{S}=J_{2} d \varphi_{2} /\left.d n\right|_{S},
$$

and static fields $\varphi_{1,2}(\mathbf{r})$ of the vortex system outside and within the defect are determined by equations

$$
J_{1} \Delta \varphi_{1}=0 \text { when }|\mathbf{r}|>a \text { and } J_{2} \Delta \varphi_{2}=0 \text { when }|\mathbf{r}|<a \text {. }
$$

The distribution of the vortex field located at distance $R$ outside the circular region of modified properties and with boundary conditions (12) is well known. ${ }^{17}$ For a vortex with topological charge $q$, a fictitious vortex with a non-integer topological charge must be placed inside the defect region at distance $b=a^{2} / R$ from the center

$$
q_{\text {in }}=q\left(J_{2}-J_{1}\right) /\left(J_{2}+J_{1}\right)=q \sigma,
$$

and an antivortex with charge $-q_{\text {in }}$ must be placed in the center of the defect. [Consideration of the finiteness of the exchange across the boundary $\left(J_{s} \neq \infty\right)$ leads to the replacement of $q_{\text {in }} \rightarrow q_{\text {eff }}=-\left(1-f\left(J_{S}\right) q_{\text {in }}\right)$, where function $f\left(J_{s}\right)$ rapidly varies from zero to unity with increasing $J_{s}$ (Ref. 17)]. The field inside the defect is determined by a fictitious vortex with charge $q_{\text {out }}=q-q_{\text {in }}=q(1-\sigma)$ at point $\mathbf{R}$. Thus, for a vortex with $q=1$ at point $\mathbf{R}(\mathrm{R}, 0)$, fields $\varphi_{1}$ and $\varphi_{2}$ outside and inside the defect are defined by expressions

$$
\begin{gathered}
\varphi_{1}=\operatorname{arctg}(y /(x-R))+\sigma \operatorname{arctg}(y /(x-b)) \\
-\sigma \operatorname{arctg}(y /(x)), \\
\varphi_{2}=(1-\sigma) \operatorname{arctg}(y /(x-R))+\sigma \pi .
\end{gathered}
$$

To describe the scattering of a vortex pair outside the defect (a vortex with charge $q=1$ at point $\mathbf{R}_{1}\left(X_{1}, Y_{1}\right)$ and an antivortex with charge $q=-1$ at the point $\mathbf{R}_{2}\left(X_{2}, Y_{2}\right)$, let us place fictitious vortices at points $\mathbf{b}_{1}\left(x_{1}, y_{1}\right)$ and $\mathbf{b}_{2}\left(x_{2}, y_{2}\right)$ inside the defect region (see Fig. 1). All the vortices are assumed to have one polarization $p_{i}=1$. Fictitious vortices at the origin are compensated, and the problem reduces to finding the field of two actual and two fictitious vortices.

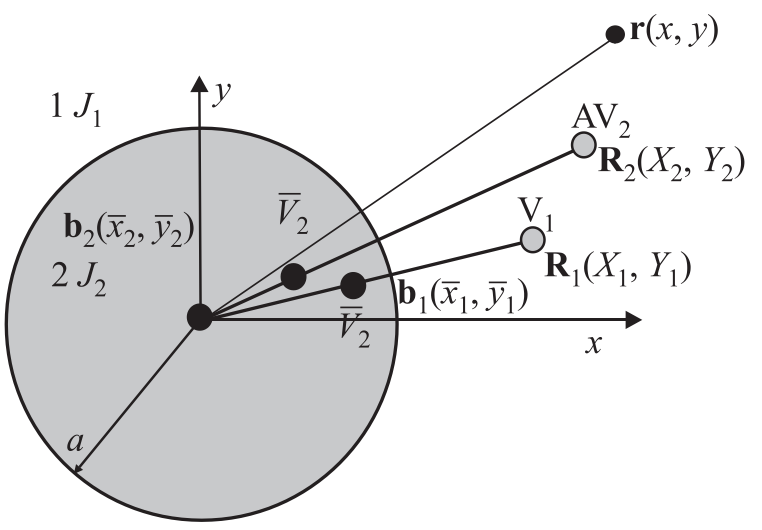

Fig. 1. Distribution of actual and fictitious vortices in the problem of a vortex pair in a system with a magnetic defect. 
Motion integrals $E$ and $K$ make it possible to solve the problem of vortex pair dynamics. Positions and charges of virtual vortices are expressed as follows

$$
\begin{gathered}
b_{i}=a^{2} / R_{i}, \quad x_{i}=a^{2} X_{i} / R_{i}^{2}, \quad y_{i}=a^{2} Y_{i} / R_{i}^{2}, \\
q_{1}=\sigma, \quad q_{2}=-\sigma .
\end{gathered}
$$

Accordingly, when determining the field inside the defect, it is necessary to place virtual vortices with charges $\tilde{q}_{1}=1-\sigma$ and $\tilde{q}_{2}=\sigma-1$ at the locations of actual vortices $\mathbf{R}_{1}$ and $\mathbf{R}_{2}$. Thus, the fields outside and inside the defect are defined as follows

$$
\begin{gathered}
\varphi_{\text {out }}=\operatorname{arctg} \frac{y-Y_{1}}{x-X_{1}}-\operatorname{arctg} \frac{y-Y_{2}}{x-X_{2}} \\
+\sigma \operatorname{arctg} \frac{y-y_{1}}{x-x_{1}}-\sigma \operatorname{arctg} \frac{y-y_{2}}{x-x_{2}}, \\
\varphi_{\text {in }}=(1-\sigma) \operatorname{arctg} \frac{y-Y_{1}}{x-X_{1}}-(1-\sigma) \operatorname{arctg} \frac{y-Y_{2}}{x-X_{2}} .
\end{gathered}
$$

"External fields" $\varphi_{i, \text { ext }}$, acting on each vortex of the pair are determined by the fictitious vortices and the field of the "complementary" vortex of the pair from the sum (18). Knowledge of these fields is sufficient for constructing the dynamics of the initial vortex pair in the framework of Eq. (10).

\section{Dynamics of an isolated vortex in the magnetic defect field}

Let us consider the dynamics of an isolated vortex with $q=p=1$ in the defect field in the proposed model. The magnetization pattern is determined by formulas (15) and (16). It follows from Ref. 7 that the vortex rotates around the defect in a circular orbit with radius $R$, which determines the energy and momentum of the system

$$
\begin{gathered}
E_{V}=\pi J_{1} M_{0}^{2} \ln \left(l / d_{0}\right)-\pi J_{1} M_{0}^{2} \sigma \ln \left(1-a^{2} / R^{2}\right), \\
K_{V}=\left(\hbar M_{0} / 2 \mu_{0}\right) \pi R^{2} .
\end{gathered}
$$

The second term in Ref. 20 describes the interaction of a vortex with a defect, qualitatively coinciding with the one given by Pereira et al. ${ }^{12}$ With $R \gg a$, the interaction energy

$$
E_{\mathrm{int}} \approx \pi J_{1} M_{0}^{2} \sigma a^{2} / R^{2}=J_{1} M_{0}^{2} \delta / R^{2}
$$

where the defect characteristic $\delta=\sigma \pi a^{2}$ is proportional to its area and varies from $\pi a^{2}$ for a non-magnetic defect to $\pi a^{2}$ for a "hard magnetic" defect. The first limit is of particular interest since it also describes the situation with a superfluid liquid with an impermeable region. In the case of a large defect area and the vortex moving along the boundary at distance $\Delta$ from it with $a \gg \Delta \gg l_{0}$, it follows from Ref. 20 that $E_{\mathrm{int}} \approx-\pi J_{1} M_{0}^{2} \sigma \ln (\Delta / a)$, which corresponds to the motion along the half-space boundary with velocity $V=J_{1} M_{0}^{2} \mu_{0} \sigma /$ $\hbar \Delta$. With a finite radius of the defect, it follows from Eq. (7) that the rotational speed of the vortex can be expressed as

$$
V=-J_{1} M_{0} \frac{2 \mu_{0}}{\hbar} \sigma \frac{a^{2}}{R\left(R^{2}-a^{2}\right)} .
$$

The vortex is "captured" by a defect and rotates around it with velocity $\propto 1 / R^{3}$ at large distances. The direction of rotation depends on the properties of the defect. As the exchange decreases compared to the matrix exchange (in particular, for a nonmagnetic defect), the vortex rotates counterclockwise, and in the case of a larger exchange, it rotates clockwise.

\section{Motion of the vortex pair in the presence of a magnetic defect}

We now turn to the study of vortex pair dynamics in the case of a defect (Fig. 2). (In the proposed model, the defect does not generate its own field when no vortices are present). The vortex pair before scattering $(t=-\infty)$ has dimension $L_{0}$ and "impact parameter" $\rho$. Let us find the dependence of the scattering angle of pair $\chi=\chi\left(\rho, L_{0}\right)$ on the impact parameter and pair dimension $L_{0}$. Dimension $L_{0}$ determines the energy of the system, and together with $\rho$, the angular momentum, i.e., two motion integrals. Below, $\mathbf{R}_{1}\left(\mathrm{X}_{1}, Y_{1}\right)$ is the coordinate of a vortex with charge $q=1$, and $\mathbf{R}_{2}\left(\mathrm{X}_{2}, Y_{2}\right)$ is the coordinate of an antivortex with charge $q=-1$. The angle between vectors $\mathbf{R}_{1}$ and $\mathbf{R}_{2}$ is denoted as $\varphi$. Positions of fictitious vortices are determined by the position of the vortices of the pair, and the problem only reduces to the motion of these two quasiparticles. Accordingly, the dynamical system with two degrees of freedom and two independent integrals of motion is integrated in quadratures. Knowledge of the magnetization field (18) outside the defect makes it possible, through (10), to write the equations of motion of the vortices within the pair. Let us introduce a new time variable $\tau=\left(2 J_{1} M_{0} \mu_{0} / \hbar\right) t$, in terms of which Eq. (10) can be rewritten as $\mathbf{R}_{\tau}=-\nabla \varphi_{\mathrm{ex}}$ or

$$
\begin{aligned}
\frac{d X_{1,2}}{d \tau}= & \frac{Y_{2}-Y_{1}}{R_{1}^{2}-2 \mathbf{R}_{1} \mathbf{R}_{2}+R_{1}^{2}} \pm \sigma \frac{Y_{1,2}}{\left(R_{1,2}^{2}-a^{2}\right)} \\
& \mp \sigma \frac{Y_{1,2} R_{2,1}^{2}-Y_{1,2} a^{2}}{R_{1}^{2} R_{2}^{2}-2 a^{2} \mathbf{R}_{1} \mathbf{R}_{2}+a^{4}}, \\
\frac{d Y_{1,2}}{d \tau}= & \frac{X_{1}-X_{2}}{R_{1}^{2}-2 \mathbf{R}_{1} \mathbf{R}_{2}+R_{2}^{2}} \mp \sigma \frac{X_{1,2}}{\left(R_{1,2}^{2}-a^{2}\right)} \\
& \pm \sigma \frac{X_{1,2} R_{2,1}^{2}-X_{2,1} a^{2}}{R_{1}^{2} R_{2}^{2}-2 a^{2} \mathbf{R}_{1} \mathbf{R}_{2}+a^{4}},
\end{aligned}
$$

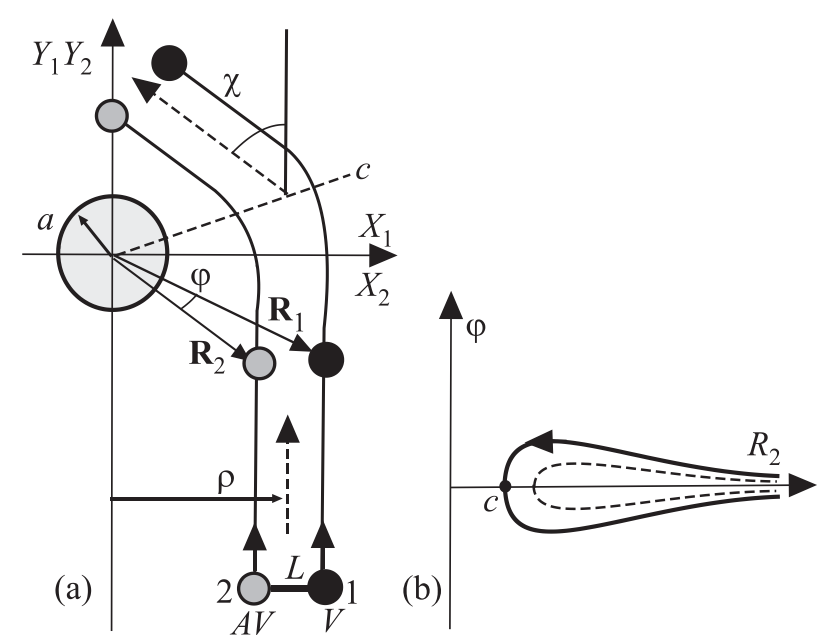

Fig. 2. Scattering of a vortex-antivortex pair on a circular ferromagnetic defect in the case of a low magnetic defect with $J_{2}<J_{1}$. Vortex pair trajectories (a) and corresponding "phase portrait" (b). 
where $R_{i}^{2}=X_{i}^{2}+Y_{i}^{2}$ and $\mathbf{R}_{1} \mathbf{R}_{2}=X_{1} X_{2}+Y_{1} Y_{2}$. These equations admit analytical solution in the small-angle scattering approximation as well as the study by the methods of the qualitative theory of dynamical systems, and numerical investigation in a wide range of values of $L_{0}, \rho$ and $\sigma$. Equations (23) and (24) coincide with those obtained in the Thiele approach from formulas (7) with the knowledge of the total energy of the system. It can be easily obtained by formulas (18) and (19), and an expression for energy (4) in the basic approximation with $m=0$

$$
\begin{aligned}
E= & 2 \pi J_{1} M_{0}^{2} \ln \frac{R_{12}}{d_{0}} \\
& +\pi J_{1} M_{0}^{2} \sigma \ln \left(\frac{R_{1}^{2} R_{2}^{2}-2 R_{1} R_{2} a^{2} \cos \varphi+a^{4}}{\left(R_{1}^{2}-a^{2}\right)\left(R_{2}^{2}-a^{2}\right)}\right),
\end{aligned}
$$

where $R_{12}(t)=\left|\mathbf{R}_{1}-\mathbf{R}_{2}\right|=\sqrt{R_{1}^{2}-2 R_{1} R_{2} a^{2} \cos \varphi+R_{2}^{2}}$ is the distance between the vortices of the pair, which is different from the initial value of $L_{0}$ and depends on time. The first term in (25) corresponds to the intrinsic energy of the pair (11), and the factors $\left(R_{1}^{2}-a^{2}\right)$ and $\left(R_{2}^{2}-a^{2}\right)$ in the second term correspond to the interaction of each vortex of the pair with the defect (see Ref. 20) The total energy includes an additional term associated with the interaction of the vortices of the pair "through the defect." The argument of the logarithm in the second term of (25) is greater than unity; with $\sigma$ $<0$, the low magnetic defect attracts the pair; with $\sigma>0$, the hard magnetic defect repels it.

The angular momentum of the pair is expressed through the initial data and is equal to

$$
K=\left(\pi \hbar M_{0} / 2 \mu_{0}\right)\left(R_{1}^{2}-R_{2}^{2}\right)=\left(\pi \hbar M_{0} / \mu_{0}\right)\left(L_{0} \rho\right) .
$$

Knowledge of motion integrals $E$ and $K$ makes it possible to study the dynamics of the vortex pair using qualitative analysis on the "phase plane" which can be conveniently represented by the distance of the antivortex (closely adjacent to the defect) to center $R_{2}$ and angle $\varphi$. From (25) and (26) in the small-angle scattering approximation with $\rho \gg a, L_{0}$, it follows that

$$
\varphi \approx\left(L_{0} / R_{2}^{2}\right) \sqrt{\left(R_{2}-\rho+L_{0} / 2\right)\left(R_{2}+\rho\right)+\left(a L_{0} / R_{2}\right)^{2}},
$$

which provides an insight into the dynamics of the system on plane $\varphi, R_{2}$. The corresponding phase trajectory is shown in Fig. 2(b), where the dotted line depicts the trajectory for the rectilinear motion of the vortex pair in the absence of a defect $(a=0)$, and point $c$ corresponds to the trajectory point closest to the defect [line $c$ in Fig. 2(a)].

\section{Small-angle scattering of vortex pairs}

Small-angle scattering is easy to investigate analytically. As variables, let us introduce the coordinates of the center of the vortex pair $\mathbf{r}=(x, y)$ and the vector of "vortex dipole" $\mathbf{d}=2 \mathbf{b}$, which determines the dimension of pair $R_{12}(t)$ $=L(t)=2 b$

$$
\begin{aligned}
& x=\left(X_{1}+X_{2}\right) / 2, \quad y=\left(Y_{1}+Y_{2}\right) / 2, \\
& u=b_{x}=\left(X_{1}-X_{2}\right) / 2, \quad v=b_{y}=\left(Y_{1}-Y_{2}\right) / 2 .
\end{aligned}
$$

The new variables are shown in Fig. 3 , where $\chi$ denotes the scattering angle. In the new variables, dynamic equations (23) and (24) are given by

$$
\begin{gathered}
{\left[\mathbf{r}_{\tau}, \mathbf{n}\right]=\mathbf{b} / 2 b^{2}+(f-s) \mathbf{b}+h \mathbf{n},} \\
{\left[\mathbf{b}_{\tau}, \mathbf{n}\right]=-s \mathbf{r}+h \mathbf{b},}
\end{gathered}
$$

where $\mathbf{n}$ is the unit vector perpendicular to the plane of the system, and the coefficients of the equations only depend on the moduli of vectors $\mathbf{r}(\tau)$ and $\mathbf{b}(\tau)$

$$
\begin{gathered}
h=\left(\sigma a^{2}\right) 8 b^{2} L_{0} \rho / P Q, \quad f=\left(\sigma a^{2}\right) 2 / Q, \\
s=\left(\sigma a^{2}\right) 4 b^{2}\left(r^{2}+b^{2}-a^{2}\right) / P Q, \\
P=\left(r^{2}+b^{2}-a^{2}\right)^{2}-\left(L_{0} \rho\right)^{2}, \quad Q=P+4 a^{2} b^{2} .
\end{gathered}
$$

For small-angle scattering, one can use the separation of time scales: the "rapid" motion of the pair center [coordinates $(x, y)]$ with velocity $V \sim 1 / L$ and the "slow" change in its shape and direction of motion [coordinates $(u, v)]$. All the terms of the equations, except the first on the right-hand side (29), have factors $\sigma a^{2} \sim \delta$ characterizing the "capacity" of the defect. The terms on the right-hand side (29) have the following orders of magnitude (left to right): $1 / b,\left(\sigma a^{2}\right) b / \rho^{4}$, $\left(\sigma a^{2}\right) b^{3} / \rho^{6},\left(\sigma a^{2}\right) b^{3} / \rho^{6}$, and on the right-hand side (30)$\left(\sigma a^{2}\right) b^{2} / \rho^{5}$ and $\left(\sigma a^{2}\right) b^{4} / \rho^{7}$. Therefore, the deviation of the pair trajectory from the linear path is small, with $\rho \gg a b$ $\sim a L_{0}$. In the basic approximation, from (29) we obtain a solution for the free motion of a pair with dimension $L_{0}$ : $\mathbf{r}_{0}=\left(\rho, \tau / L_{0}\right)=\left(\rho, t\left(J_{1} M_{0} \mu_{0} / \hbar L_{0}\right)\right)$ and $r_{0}=\sqrt{\rho^{2}+\tau^{2}} / L_{0}^{2}$, which agrees with solution (11) (dotted lines in Fig. 3), and in this approximation $b=b_{0}=u_{0}=L_{0} / 2, v_{0}=0$. For a small pair with $L \ll r$, i.e., with $b \ll \rho$, Eq. (30) takes the form of $\left[\mathbf{b}_{\tau}, \mathbf{n}\right]=-s \mathbf{r}$, or when taking into account the type of the basic approximation: $v_{\tau}=-s \rho$ and $u_{\tau}=s \tau / L_{0}$, where $s \approx \sigma a^{2} L_{0}^{2} /\left(\rho^{2}+\tau^{2} / L_{0}^{2}\right)^{3}$. The integration gives the asymptotics of the vortex pair reversal with $\tau \rightarrow \pm \infty: v( \pm \infty)$ $=\mp\left(\pi a^{2} \sigma\right) 3 L_{0}^{3} / 16 \rho^{4}$. The angle of its rotation for half the scattering time is equal to $\Delta \approx 2 v / L_{0}$ (Fig. 3), and the total scattering angle $\chi=2 \Delta$. Therefore, dependence $\chi=\chi(\rho)$ is defined by the following formula

$$
\chi\left(\rho, L_{0}\right)=3 \delta L_{0}^{2} / 4 \rho^{4} .
$$

From the second equation for the pair dipole, we find a change in its size during the scattering of $L=L_{0}\left(1-\sigma a^{2} L_{0}^{2} /\right.$ $\left.\rho^{4}\right)$ : when the pair is repelled from the defect $(\sigma>0)$, it

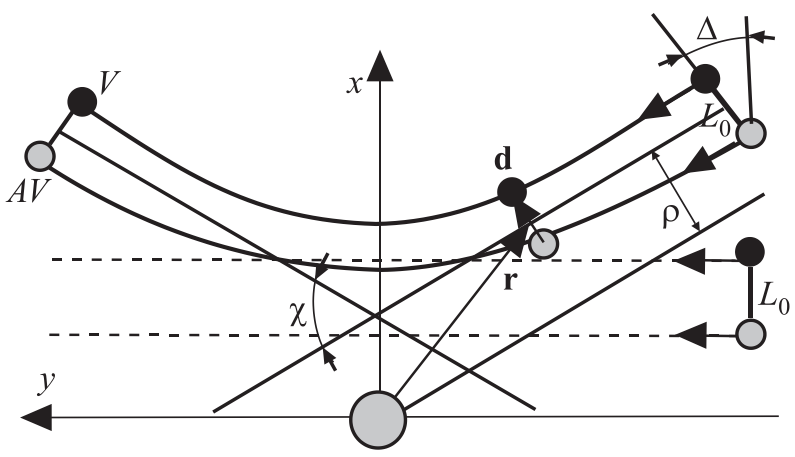

Fig. 3. Low-amplitude scattering of a vortex pair (hard magnetic impurity). 
shrinks in the interaction area and it stretches out when attracted to the defect $(\sigma<0)$.

Similarly, the obtained result follows from the formula for the low-amplitude scattering of ordinary particles, ${ }^{18}$ if we rewrite it as

$$
\chi=-\left(2 \rho / P_{0} V_{0}\right) \int_{\rho}^{\infty} d r(d U / d r) / \sqrt{\rho^{2}-r^{2}},
$$

where the momentum and velocity of the vortex pair at infinity (11) are equal to $P_{0}=\pi \hbar M_{0} L_{0} / \mu_{0}$ and $V_{0}=2 J_{1} \mu_{0} M_{0} /$ $\hbar L_{0}$, and the potential energy is equal to the total energy of the vortex pair in defect field (25), which reduces in the considered limit to $E \approx\left(\sigma \pi a^{2}\right) J_{1} M_{0}^{2} L_{0}^{2} / r^{4}$. Recently, methods have been developed for the generation of vortices and vortex pairs in BEC, as well as vortices and skyrmions in magnets under the action of a high-frequency field and under laser and thermal effects. ${ }^{13,19}$ The associated generation of a great number of vortex excitations and their directed motion cause problems in terms of averaging the results, ${ }^{20}$ and it is of interest to calculate the differential scattering cross section of the vortices and vortex pairs on the ensemble of defects. Calculated in the usual way ${ }^{18}$ for a two-dimensional case, this quantity is equal to

$$
\sum(\rho)=|d \rho / d \chi| d \chi=(3 \pi \sigma)^{1 / 4} \sqrt{a L_{0} / 32} \chi^{-5 / 4} d \chi
$$

\section{Scattering process for arbitrary parameters of vortex pairs and defects}

Although the small-angle scattering makes the main contribution to the differential scattering cross section, we find it interesting to study this process for arbitrary relations of the task parameters: $a, \sigma, L_{0}$ and $\rho$. Scattering in the general case was considered numerically in the framework of Eqs. (23) and (24) using the MAPLE software. Scattering angle $\chi$ is determined using only dimensionless combinations of parameters $L_{0}, a$ and $\rho$. The method of collective variables implies the inequality $L_{0}, \Delta \gg l_{0}$, where $\Delta$ is the smallest distance from the vortices to the boundary of the defect. Despite the fact that the conditions for the applicability of Eqs. (23) and (24) are violated as to critical parameter ratios when the vortices move sufficiently close to the boundaries of the defect $\left(\Delta \ll l_{0}\right)$, the obtained results qualitatively give a general characteristic of the dynamics of the "vortex pair-defect" system. The nature of scattering is different for repulsive (for $\sigma>0$ ) and attractive (for $\sigma<0$ ) defects. Parameter $a$ varies in the interval of $-1<\sigma<1$. Let us present the results for the most characteristic cases $\sigma=1$ and $\sigma=-1$. The latter is of particular interest, as the data for the magnetic system are then transferred to the important problem of the interaction of vortex pairs with a solid inclusion in a superfluid liquid.

\subsection{Scattering of pairs by an attractive (non-magnetic) defect}

As an example, Fig. 4(a) shows numerical results for the dependence of scattering angle $\chi$ on impact parameter $\rho$ in limit $\sigma=-1$ for fixed values of defect radius $a=2$ and pair dimension $L_{0}=1$. It varies significantly with a critical value of impact parameter $\rho=\rho_{c} \approx 3.4$. Limit $\rho \gg \rho_{c}$ corresponds
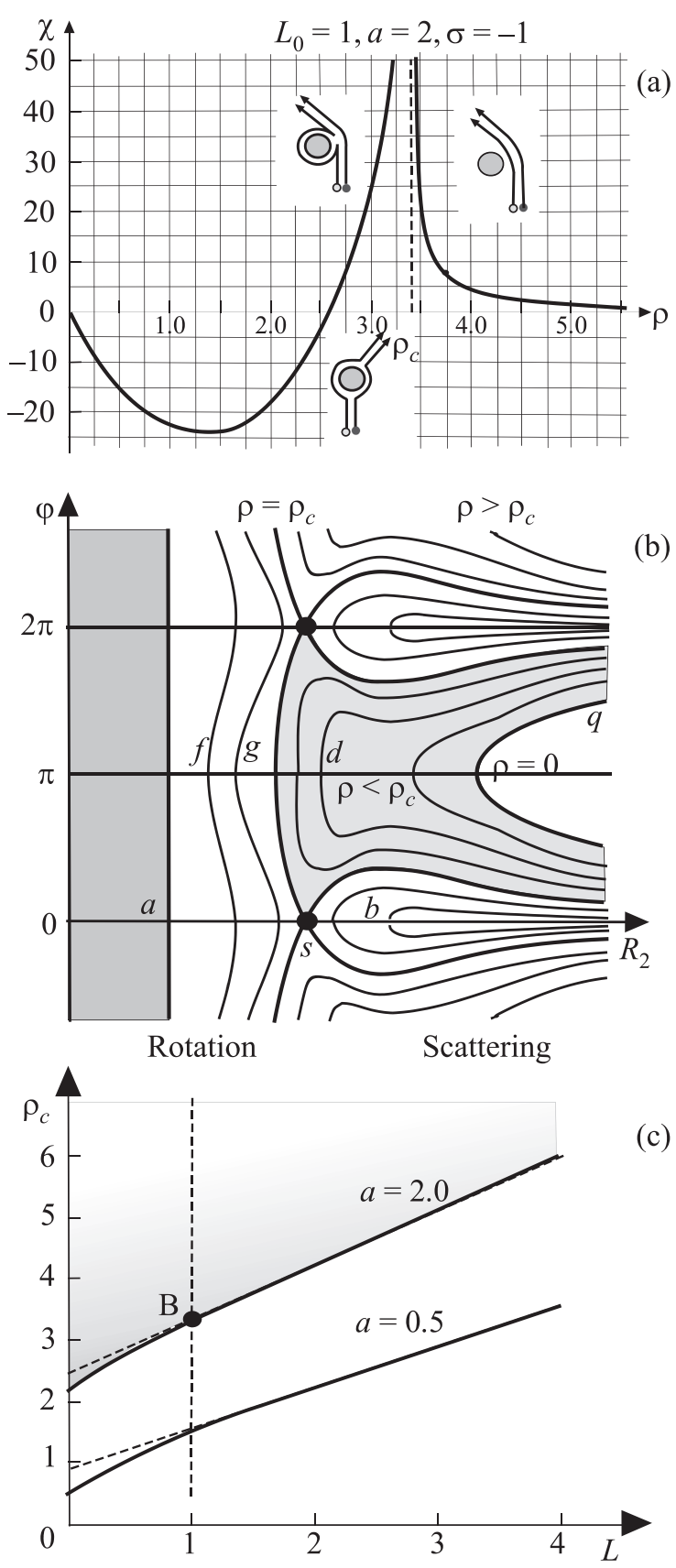

Fig. 4. Dependence $\chi=\chi(\rho)$ with $L_{0}=1$ and $a=2$ (a), the "phase portrait" of the vortex pair scattering on plane $\varphi, R_{2}$ with different values of $\rho$ (b), dependence $\rho_{c}=\rho_{c}(L)(\mathrm{c})$.

to the small-angle scattering discussed above; however, with $\rho=\rho_{c}$, the scattering scenario changes. When $\rho>\rho_{c}$, both vortices of the pair envelop the defect on one side [Fig. 2(a)]; when $\rho<\rho_{c}$, then, in the process of scattering, the pair decays into two vortices that bypass the obstacle from different directions, and again restores itself as a single object after passing through the defect [Fig. 5(a)]. When $\rho<\rho_{c}$ and $\rho \rightarrow \rho_{c}$, the trajectory of the antivortex moving near the defect surface changes dramatically [Fig. 5(b)]: for some part of the trajectory, it travels in the direction opposite to the rotation of the pair vortex. As soon as the sign of ( $\rho-$ $\rho_{c}$ ) changes, the difference in the directional angles of vectors $\mathbf{R}_{1}$ and $\mathbf{R}_{2}$ changes abruptly by $2 \pi$. As $\rho \rightarrow 0$, the distance of the vortices to the defect boundary tends to $\Delta \approx L_{0}$ / 2 , since the energy of the vortex pair is distributed almost equally between two pairs of vortices with their images 
(a)

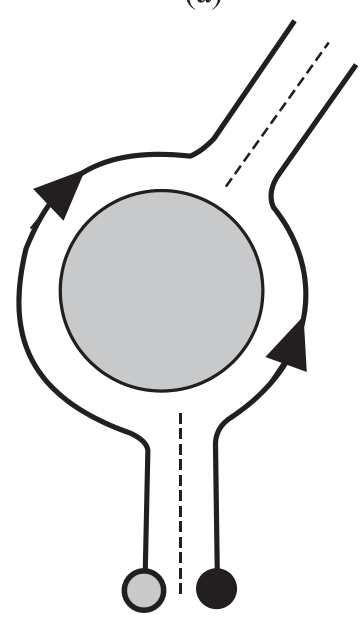

(b)

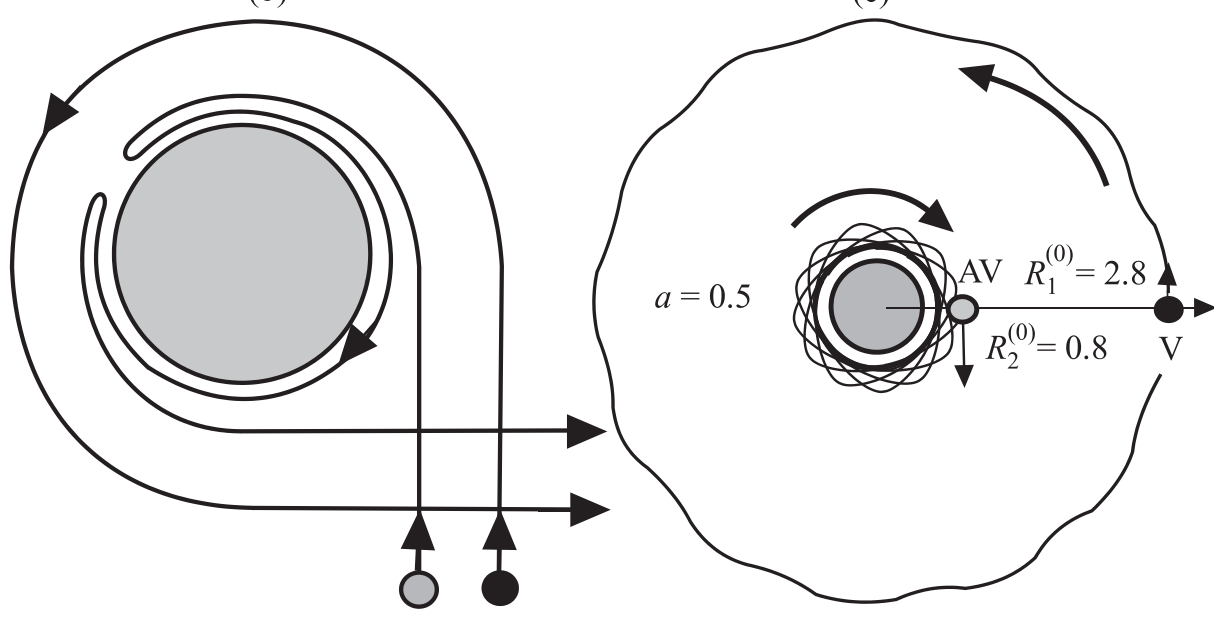

Fig. 5. Dependence of the scattering pattern of the vortex pair with $\rho<\rho_{c}$ for different dimensional ratios of the pair and the defect: $L_{0}=2, a=0.5, \rho \ll \rho_{c}$ (a) and $L_{0}=1, a=0.5, \rho \approx \rho_{c}$ (b). Rotation pattern of the vortex pair around the defect at $a=2, M=7.2, L \approx 2, \rho=M / 2 L_{0} \approx 1.8$ (c).

[Fig. 5(a)]. These results are qualitatively consistent with those given in Ref. 13 for the repulsive impurity in BEC. (The repulsive impurity in $\mathrm{BEC}$ corresponds to the attractive magnetic defect).

A clear idea of the scattering nature is provided by its analysis on "phase plane" $\varphi, R_{2}$. For $\sigma=-1$, expression (25) implies dependences $\varphi=\varphi\left(R_{2}\right)$ for different impact parameters $\rho$ and energies of the vortex pair $E=2 \pi M_{0}^{2} \ln \left(L_{0} / d_{0}\right)$ :

$\sin ^{2}\left(\frac{\varphi}{2}\right)=\frac{L_{0}^{2}\left(R_{1} R_{2}-a^{2}\right)^{2}-\left(R_{1}-R_{2}\right)^{2}\left(R_{1}^{2}-a^{2}\right)\left(R_{2}^{2}-a^{2}\right)}{4 R_{1} R_{2}\left(\left(R_{1}^{2}-a^{2}\right)\left(R_{2}^{2}-a^{2}\right)-a^{2} L_{0}^{2}\right)}$.

Since $R_{1}=\sqrt{R_{2}^{2}+2 \rho L_{0}}$, expression (32) defines dependence $\varphi=\varphi\left(R_{2}, \rho, L_{0}\right)$. It is qualitatively shown in Fig. 4(b), where the shaded area with $R_{2}<a$ corresponds to the defect area. As coordinate $R_{2}$ of the antivortex approaches a small distance to this boundary, the conditions for applicability of the analysis are violated; however, we shall use the results obtained for a qualitative understanding of the scattering process.

The areas $\rho>\rho_{c}$ in Fig. 4(b) correspond to type (b) "trajectories" for which, before and after scattering, the angle between the radius vectors of the vortices is equal to zero. When $\rho<\rho_{c}$, the scattering process corresponds to the shaded area $(d)$ of the trajectories for which the angle between the radius vectors changes during scattering by $2 \pi$ (i.e., one of the paired vortices "envelops" the defect). In the case of "end-on" collision at $\rho=0$ (boundary line $q$ in the figure), the vortices bypass the defect from different sides with a minimal distance to its boundary $R_{0}$, which depends on relation $l=L_{0} / 2 a$ and for $z=R_{0} / a$ is defined by equation $z^{3}-l z^{2}-z-l=0: R_{0} \approx L_{0} / 2$ at $L_{0} \gg a$ and $R_{0} \approx a$ $+L_{0} / 2$ for $L_{0} \ll a$. "Phase trajectories" of type $(d)$ close to the separatrix $(g)$ correspond to actual trajectories such as those shown in Fig. 4(c). In a certain time interval, the vortices of the pair rotate in the opposite direction, and this interval increases in limit $\rho \rightarrow \rho_{c}$. "Saddle point" $s$ of the phase portrait corresponds to value $\rho=\rho_{c}$. It follows, from Eqs. (23) and (24), that this point corresponds to the in-phase rotation of the vortex pair around the defect. However, this motion is unstable, which is typical for saddle points, and vortices (see Fig. below) begin to rotate in opposite directions. From Eq. (32) at $\varphi=0$, it follows that, at the critical point for large dimensions of the pair $\left(L_{0}>a\right)$, we have $\rho_{c} \approx L_{0} / 2+3 a^{2 / 3} L_{0}^{1 / 3}$ [Fig. 4(c)]. At the limit of $R_{2} \approx a^{2 / 3}$ $L_{0}^{1 / 3} \gg a$, the condition of applicability of the equation is fulfilled. Figure 4(c) shows dependence $\rho_{c}=\rho_{c}\left(L_{0}\right)$ derived numerically for $a=2$. The impact parameters under which the pair does not break apart near the defect are shaded. In Fig. 4(a), point $B$ marks a peculiarity, and a vertical dashed line marks a breakdown along the axis of impact distances. Dependence $\rho_{c}=\rho_{c}\left(L_{0}\right)$ is practically linear across the entire range of changes in the impact distance, except for the area of small impact parameters of the order of quantities $L_{0}$ and $a$. Dependence $\rho_{c}=\rho_{c}\left(L_{0}\right)$ for $a=0.5$ is additionally provided for comparison. The results of the analysis indicate that critical value $\rho_{c}$ at $\sigma=-1$ exists for any values of the pair and defect dimensions.

The analysis of the phase portrait indicates that there is an area of completely different dynamics [line $(f)$ in Fig. 4(b)] for values $R_{2}$ that are smaller than the separatrix $(g)$. It corresponds to rotation of the vortex pair near the defect. Parameter $L_{0}$ no longer denotes the dimension of the pair when it approaches infinity, but remains a characteristic of the system energy. In this case, the vortex and the antivortex rotate in the opposite direction with different velocities. Their motion is two-frequency and occurs with two incommensurable frequencies in the general case. A typical form of this rotation, which was obtained numerically, is shown in Fig. 5(c) for the following initial conditions: $R_{1}(t=0)=2.8$, $R_{2}(t=0)=0.8, M(t=0)=R_{1}^{2}-R_{2}^{2}=7.2$.

\subsection{Scattering of pairs by a repulsive defect}

When $\sigma>0$ ("hard magnetic" defect), a vortex pair repels from it and its scattering varies significantly. Let us study the limit $\sigma=1\left(J_{2} \rightarrow \infty\right)$ and consider the scattering process on the "phase plane" $\varphi, R_{2}$. In this case, instead of relation (32), we have connection $\varphi\left(R_{2}\right)$ as follows 


$$
\begin{aligned}
\cos \varphi= & \frac{1}{4 a^{2} R_{1} R_{2}}\left(\left(R_{1}^{2}+a^{2}\right)\left(R_{2}^{2}+a^{2}\right)\right. \\
& -\sqrt{\left(R_{1}^{2}-a^{2}\right)\left(R_{2}^{2}-a^{2}\right)} \\
& \left.\times \sqrt{\left(R_{1}^{2}-a^{2}\right)\left(R_{2}^{2}-a^{2}\right)+4 a^{2} L_{0}^{2}}\right),
\end{aligned}
$$

where $R_{1}=\sqrt{R_{2}^{2}+2 \rho L_{0}}$. It describes the scattering process for different values of $\rho, L_{0}$, and $a$, and is shown in Fig. 6(b). A numerical solution of Eqs. (23) and (24) for a large-size pair at $L_{0}=6 \gg a=0.5$ gives the dependence of the scattering angle on the impact parameter, as shown in Fig. 6(a), which resembles the dependence in the case of an attracting defect with the changing sign $\left(\rho-\rho_{c}\right)$. There is also a critical value $\rho_{c}$ of the impact parameter at which the scattering pattern changes, but now it only exists at sizes of the pair that
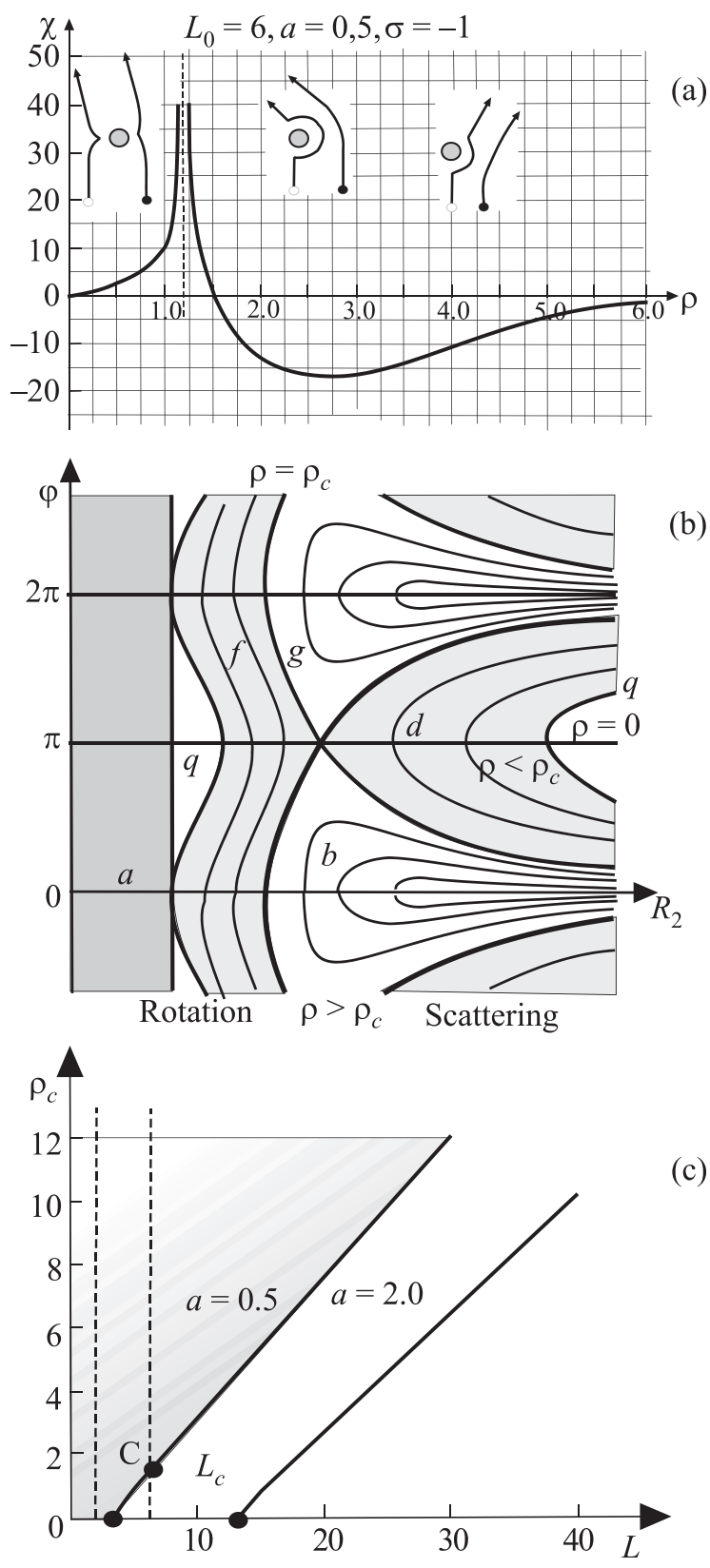

Fig. 6. Dependence $\chi=\chi(\rho)$ with $L_{0}=6$ and $a=0.5$ for the repulsive defect (a), the "phase portrait" of the scattering dynamics of the pair (b), and dependence of the critical impact distance on the dimensions of the vortex pair and the defect (c). The inserts (a) show qualitatively the trajectories of the vortices in the scattering process. are larger than the critical size $L_{c}=a(1+\sqrt{5}) \sqrt{2+\sqrt{5}}$ $\approx a \cdot 6.66$, depending on the size of the defect [Fig. 6(c)]. For a fixed pair size $L>L_{c}(a)$, the scattering process changes with a change in the impact distance. When $\rho>\rho_{c}$ $(L, a)$, both vortices of the pair remain on the same side of the defect throughout the process, which corresponds to type (b) lines in Fig. 6(b). When $\rho=\rho_{c}$ [line ( $g$ ) in Fig. 4(b)], a bifurcation takes place, breaking the pair into two vortices that bypass the defect from different sides. With further decrease of $\rho$, the vortices envelop the defect and the relative angle between them changes by $2 \pi$ [type $(d)$ lines] in the scattering process.

In an end-on collision ( $\rho=0$ and line $q$ in the figure), the minimum distance from the vortices to the defect surface depends on the size of the pair. When $L_{0} \gg a$, we have $R_{2}^{\min } \approx L_{0} / 2$, whereas for $L_{0} \ll a$, a minimal approach to the defect is $R_{2}^{\min }-a \approx L_{0} / 2$. The phase portrait also shows the possibility of a two-frequency rotation of the vortex pair around the defect (shaded area $f$ near the surface of the defect).

The situation changes for vortex pairs of small size when $L<L_{c}$ (a) [left vertical dashed line in Fig. 6(c)]. They do not pass through the defect, and are instead reflected from it. As the impact distance decreases, the scattering angle increases from zero to $\pi$, and with $\rho \rightarrow 0$, the minimum distances of the vortices to the defect surface tend to zero: $R_{2}^{\min } \approx a+\rho^{2} L_{0} / 2 a^{2}$ and $R_{1}^{\min } \approx a+\rho L_{0} / a$. (However, it should be recalled that, in this case, the applied technique of collective variables becomes invalid since the velocities of the vortices increase indefinitely, exceeding the velocity of the ferromagnet with spin waves).

\section{Conclusion}

In the approximation of the collective variables technique, the scattering of magnetic vortex pairs by a magnetic defect in a two-dimensional easy-plane ferromagnet was analyzed analytically, numerically, and qualitatively. A defect model was proposed in the form of a circular region of a magnet with an exchange interaction that differs from that in the bulk of the magnet and varies from zero to infinity. The motion of single vortices and vortex pairs in the neighborhood of the defect was studied. For the small-angle scattering, the dependence of the scattering angle of the vortex pair on the impact distance was analytically obtained and the differential scattering cross-section was calculated. With an arbitrary relation of the parameters of the vortex pair and the defect, the dependence of the scattering angle on the impact distance was numerically obtained and investigated by qualitative methods on the phase plane. In the case of a nonmagnetic impurity, the results agree qualitatively with the data from a numerical analysis of the interaction of vortex pairs with defects in BEC. The existence of bound states of a vortex pair with a defect in the form of a two-frequency counter-rotating vortex and antivortex around the defect was indicated. In a particular case, the results describe the motion of vortices and vortex pairs in the presence of a circular impermeable region in the superfluid liquid and BEC.

This work was supported by the Scientific Project No. 4/17-N of the National Academy of Sciences of Ukraine and Scientific Program No. 1.4.10.26/F-26-4. 
a)Email: kovalev@ilt.kharkov.ua

${ }^{1}$ A. M. Kosevich, B. A. Ivanov, and A. S. Kovalev, Nonlinear Waves of Magnetization. Dynamic and Topological Solitons (Naukova Dumka, Kiev, 1988).

${ }^{2}$ A. M. Kosevich, B. A. Ivanov, and A. S. Kovalev, Phys. Rep. 194, 117 (1990).

${ }^{3}$ A. M. Kosevich, V. P. Voronov, and I. M. Manzhos, JETP 84, 148 (1983).

${ }^{4}$ A. V. Nikiforov and E. B. Sonin, JETP 58, 373 (1983).

${ }^{5}$ L. Onsager, Suppl. Nuovo Cimento 6, 249 (1949); R. P. Feynman, Phys. Suppl. 24, 18 (1958).

${ }^{6}$ M. H. Anderson, J. B. Ensher, M. R. Mattheus, C. E. Wieman, and E. A. Conell, Science 269, 198 (1995); K. B. Davis, M.-O. Mewes, M. R. Andrews, N. I. van Druten, D. S. Durfee, D. M. Kurn, and W. Ketterle, Phys. Rev. Lett. 75, 3969 (1995).

${ }^{7}$ J. Raabe, R. Pulwey, R. Sattler, T. Schweinbock, J. Zweek, and D. Weiss, J. Appl. Phys. 88, 4437 (2000).

${ }^{8}$ W. J. Kwon, J. H. Kim, S. W. Seo, and Y. Shin, Phys. Rev. Lett. 117, 245301 (2016); S. Higashitani, H. Takeuchi, S. Matsuo, Y. Nagato, and K. Nagai, ibid. 110, 175301 (2013).

${ }^{9}$ N. Papanicolaou and P. N. Spathis, Nonlinearity 12, 285 (1999); S. Komineas and N. Papanicolaou, e-print arXiv:0712,3684v1condmat.meshall; S. Komineas, Phys. Rev. Lett. 99, 117202 (2007).

${ }^{10}$ A. S. Kovalev, S. Komineas, and F. G. Mertens, Eur. Phys. J. B 25, 89 (2002); A. S. Kovalev, Low Temp. Phys. 43, 274 (2017).
${ }^{11}$ M. M. Bogdan, Low Temp. Phys. 31, 735 (2005); M. M. Bogdan and C. E. Zaspel, Phys. Status Solidi A 189, 983 (2002); G. M. Wysin, Phys. Rev. B 68, 184411 (2003); L. A. S. Mol, A. R. Pereira, and A. S. T. Pires, ibid. 66, 052415 (2003); S. A. Leonel, Pablo Zimmermann Coura, A. R. Pereira, L. A. S. Mol, and B. V. Costa, ibid. 67, 104426 (2003); A. R. Pereira, L. A. S. Mol, S. A. Leonel, P. Z. Coura, and B. V. Costa, ibid. 68, 132409 (2003).

${ }^{12}$ A. R. Pereira, L. A. S. Mol, S. A. Leonel, P. Z. Coura, and B. V. Costa, Phys. Rev. B 68, 132409 (2003).

${ }^{13}$ A. Griffin, G. W. Stagg, N. P. Pronkakis, and C. F. Barenghi, e-print arXiv:1609.06226v1cond-mat.quant-gas.

${ }^{14}$ L. D. Landau and E. M. Lifshitz, Phys. Z. Sov. 8, 153 (1935).

${ }^{15}$ A. A. Tiele, Phys. Rev. Lett. 30, 230 (1973).

${ }^{16}$ H. J. Lagt, Introduction to Vortex Theory (Vortex Flow Press, Potomac, Maryland, 1961).

${ }^{17}$ H. Fan and G. F. Wang, Mech. Mater. 35, 943 (2003), see also L. D. Landau and E. M. Lifshitz, Electrodynamics of Continuous Media (Nauka, Moscow, 1982).

${ }^{18}$ L. D. Landau and E. M. Lifshitz, Mechanics (Nauka, Moscow, 1965).

${ }^{19}$ E. C. Samson, K. E. Wilson, Z. L. Newman, and B. P. Anderson, Phys. Rev. A 93, 023603 (2016).

${ }^{20}$ D. Pinna, F. A. Arauja, J.-V. Kim, V. Cros, D. Querlioz, P. Bessiere, J. Droulez, and J. Grollier, e-print arXiv:1701.07750v1.cond-mat.mes-hall.

Translated by CWG 